\title{
An unusual manifestation of amiodarone toxicity: lung mass mimicking a lung carcinoma
}

\author{
U A Liyanage ${ }^{1}$, T Rajapakse ${ }^{1}$, S C Somaratne ${ }^{1}$, P S Gunaratne ${ }^{1}$
}

Ceylon Medical Journal 2011; 56: 74-76

\section{Introduction}

Amiodarone is a widely used antiarrythmic agent with well known toxic side effects that can involve lung, liver, thyroid and neuromuscular system. Amiodarone related pulmonary toxicity commonly presents as pneumonitis causing lung fibrosis. However, amiodarone toxicity can present as a lung mass $[1,2]$. Such lesions can mimic a lung carcinoma both on chest $\mathrm{X}$ - ray and CT.

\section{Case report}

A 65-year old man with ischaemic heart disease developed progressive weakness of the lower limbs over six months which made him bed-bound. He had features of peripheral neuropathy with grade I muscle power. The nerve conduction studies showed a demyelinating sensory motor neuropathy. Liver enzymes were elevated (AST 108 U/l, ALT 132 U/l.). Thyroid functions were normal.

The chest X-ray demonstrated an ill-defined opacity in the apex of the right lung. Contrast enhanced CT scan of the chest and abdomen revealed a spiculated, irregular, hyperdense mass lesion measuring $2.5 \mathrm{~cm}$ in diameter, in the apex of the right lung suspicious of a malignant lung mass. There were bilateral thick, linear, pleural based hyperdensities, predominantly distributed in the lung bases. A few small high density peripheral lung consolidations were also present. Liver and spleen showed diffuse, high density in the non contrast CT scan (85-90 Hu compared to 50- $60 \mathrm{Hu}$ of normal liver).

CT features of peripheral consolidations and subpleural deposits raised the radiological diagnosis of amiodarone toxicity which was supported by the findings of increased density of liver and spleen. Subsequent inquiry revealed that the patient was taking amiodarone $200 \mathrm{mg}$ three times a day for more than 10 years. He had defaulted clinic follow up for 10 years. Fine needle aspiration (FNAC) of the suspicious lung mass under CT guidance showed cytology consistent with amiodarone toxicity. Amiodarone was discontinued and patient was given limb physiotherapy.

Four months after discontinuing amiodarone, patient was able to walk with support and CT scan showed marked reduction in size of the lung mass, almost complete regression of subpleural hyperdense deposits and return of liver and splenic densities to normal levels.

${ }^{1}$ National Hospital of Sri Lanka.

Correspondence: UL, e-mail <udari8@gmail.com>. Received 21 May 2010 and revised version accepted 14 January 2011. Competing interests: none declared. 


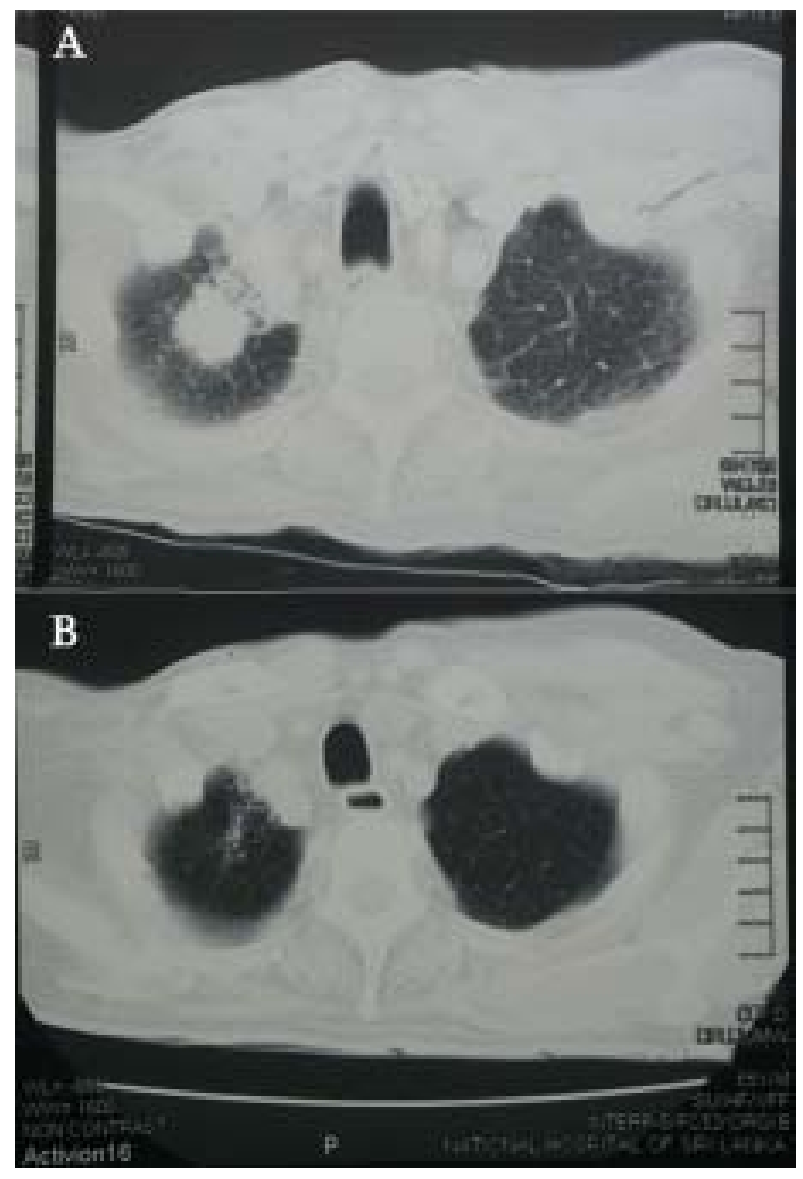

Figure 1. CT demonstrating A) $2.5 \mathrm{~cm}$ size, irregular lung mass in apex of right lung.

B) Marked regression of lung mass 4 months after withdrawing amiodarone.
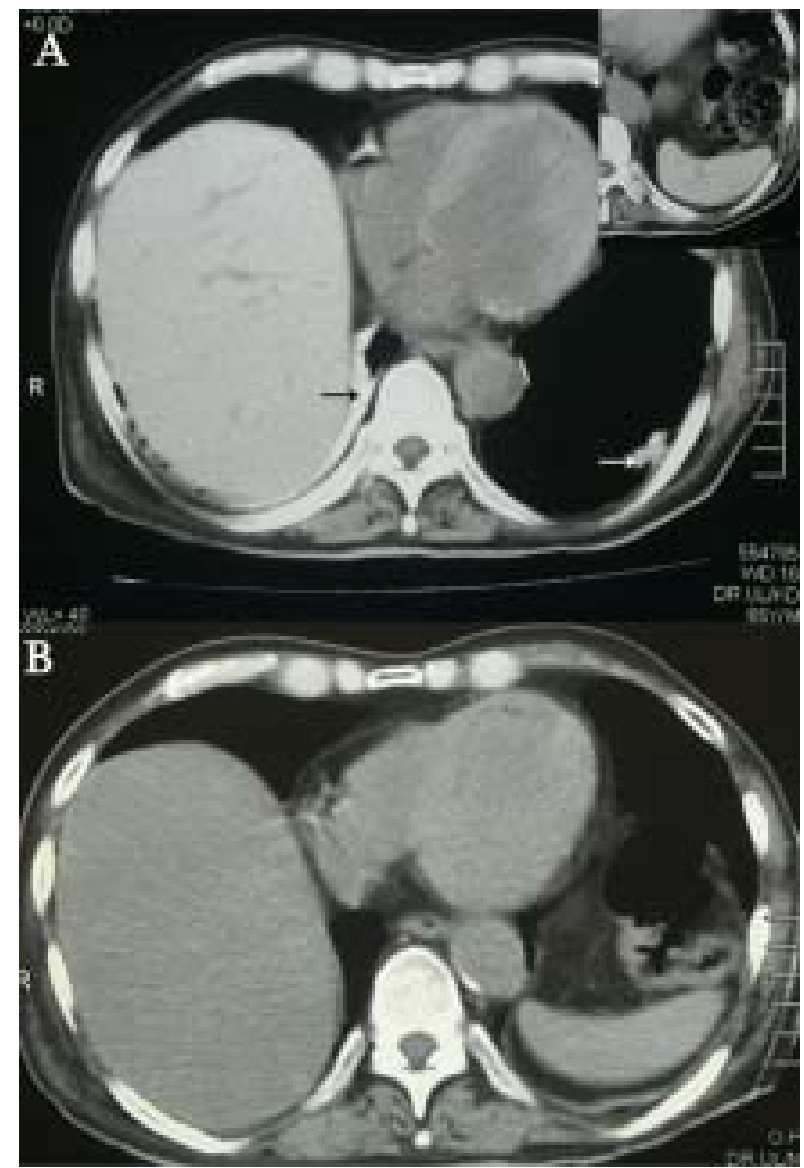

Figure 2. A) Non-contrast CT demonstrating linear subpleural hyperdensites (black arrow), increased density of liver and spleen (inset) and peripheral consolidations (white arrow).

B) CT scan 4 month after withholding amiodarone shows normal liver and splenic densities.

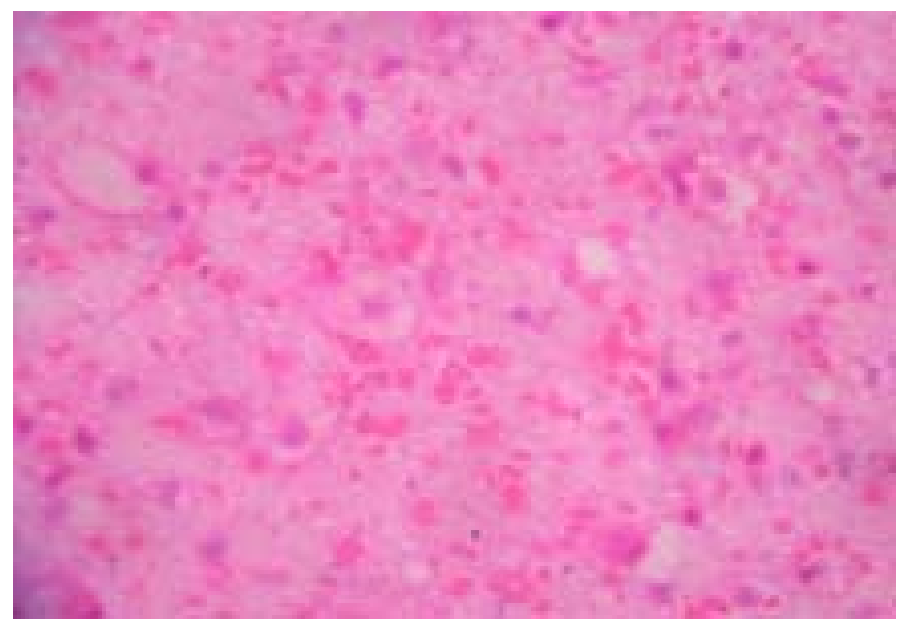

Figure 3. FNAC of lung mass showing numerous large macrophages with abundant vacuolated thin cytoplasm and background lymphocytes. (x400) 


\section{Discussion}

Amiodarone induced lung toxicity has been observed in up to $10 \%$ of patients receiving this drug [3]. Toxicity usually occurs between 3 months to 5 years.

Although chronic interstitial pneumonitis is the more frequent pulmonary manifestation amiodarone toxicity can cause a lung mass. These mass lesions, referred to as amiodaronoma by some mimick lung carcinoma. Interestingly, all previously reported cases describe such masses in upper lobes particularly in the right lung similar to our patient [3]. Histologically lesions constitute of aggregates of foamy macrophages with vacuolated cytoplasm and chronic interstitial inflammation. Spontaneous regression of lung masses occur following drug withdrawal. Peripheral neuropathy is an uncommon manifestation of amiodarone toxicity compared to pulmonary, thyroid and liver toxicity.

There are well documented CT features of amiodarone toxicity. Dense peripheral consolidations, coarse lung fibrosis subpleural deposits of amiodarone leading to linear hyperdensities and mediastinal lymphadenopathy can occur [4]. Diffuse increase in density of liver and spleen occur due to deposition of amiodarone, which has a high content of iodine.

\section{References}

1. Azzam I, Tov N, Elias N, Naschitz JE. Amiodarone toxicity presenting as pulmonary mass and peripheral neuropathy: the continuing diagnostic challenge. Postgraduate Medical Journal 2006; 82: 73-5.

2. Jarand J, Lee A, Leigh R. Amiodaronoma: an unusual form of amiodarone-induced pulmonary toxicity. Canadian Medical Association Journal 2007; 176: 1411-3.

3. Ott MC, Khoor A, Leventhal JP, Paterick TE, Burger CD. Pulmonary toxicity in patients receiving low-dose amiodarone. Chest 2003; 123: 646-51.

4. Poll Lw, May P, Koch Ja, Hetzel G, Heering P, et al. HRCT findings of amiodarone pulmonary toxicity: clinical and radiologic regression. Journal of Cardiovascular Pharmacology and Therapeutics 2001; 6: 307-11.

5. Wolkove N, Baltzan M. Amiodarone pulmonary toxicity. Canadian Respiratory Journal 2009; 16: 43-8. 